\title{
Die Komplexität der (Kultur-)Landschaft
}

\author{
Hendrikje Wehnert
}

(C) Springer-Verlag 2012

Landschaften, in denen wir leben, die wir erleben, nutzen und gestalten, planen, verwüsten, schützen - sind schön oder unattraktiv, inspirierend, anregend oder aufregend, sind vielfach genutzt oder liegen brach. Dort können sich Natur und Mensch entfalten oder sich gegenseitig behindern. Landschaften sind Lebensgrundlagen für Mensch und Natur, die - so hat es der Gesetzgeber im Bundesnaturschutzgesetz zum Ausdruck gebracht - zunehmend stärker geschützt werden müssen, um die Leistungs-, Funktionsund Regenerationsfähigkeit des Naturhaushalts sowie die Vielfalt, Eigenart und Schönheit zu erhalten.

Wie lassen sich Landschaften erfassen, gliedern und voneinander abgrenzen? Diese Frage stellen sich Natur- und Geisteswissenschaftler und auch Künstler. Es gibt unterschiedliche Sichtweisen auf den Begriff und somit eine Vielfalt an Definitionen. Ein kurzer, nicht auf Vollständigkeit abzielender Exkurs in die Historie soll aufzeigen, wie sich der Begriff der (Kultur-)Landschaft in vergangenen Jahrhunderten und Jahrzehnten entwickelt hat.

Nach Ansicht des Philosophen Joachim Ritter führten erst die Auflösung des geschlossenen christlich-metaphysischen Weltbildes sowie die zunehmende (technische) Beherrschung der Natur dazu, dieser zweckfrei gegenüberzutreten und sie zu ästhetisieren. Diesen Prozess verortet er historisch im Übergang vom Mittelalter zur Neuzeit. Im 16./17. Jahrhundert wurde das Landschaftsbild und -verständnis maßgeblich durch die europäische und hier insbesondere durch die deutsche, französische, holländische und italienische Landschaftsmalerei geprägt. Landschaft wurde als sichtbarer und vor allem darstellbarer Ausschnitt der

H. Wehnert $(\bowtie)$

Leibniz-Institut für ökologische Raumentwicklung,

Weberplatz 1, 01017 Dresden, Deutschland

E-Mail: h.wehnert@ioer.de
Erdoberfläche verstanden, charakterisiert durch eine idealisierte natürliche Erscheinung unterschiedlicher biotischer und abiotischer Landschaftskomponenten wie Relief, Flora und Fauna. Gleichwohl spielt der Mensch in seiner gestaltenden Funktion in der Landschaftsmalerei dieser Epoche eine deutlich sichtbare Rolle: geschichtlich oder architektonisch bedeutende Bauwerke bilden den zentralen Punkt in der Bildkomposition und die Landschaft bettet sich um diese herum.

Dieser Zugang zum Verständnis von Landschaft, über die Abbildung selbiger, wurde Ende des 18. und Anfang des 19. Jahrhunderts insbesondere im deutschen Kulturkreis verstärkt, auch wenn sich der Schwerpunkt dessen, was man mit Landschaft assoziierte, etwas wandelte. In der Epoche der Romantik rückte die Erhabenheit der Natur als Ausdruck göttlichen Wirkens in den Vordergrund. Der anthropogene Einfluss in der dargestellten Landschaft scheint geringer zu sein, obgleich sich auch in dieser Epoche - dezent platzierte - Bauwerke in der Landschaftsmalerei finden lassen. Es bleibt festzuhalten, dass Landschaft stets als etwas Ästhetisches, Schönes angesehen wird. Diese positiv wertende Sichtweise prägt auch über weitere Jahrhunderte hinweg die Wahrnehmung und das Selbstverständnis von Landschaft. Dieses Paradigma wird auch durch die beginnende naturwissenschaftliche Auseinandersetzung mit dem Begriff „Landschaft“ nicht infrage gestellt. Es ist der Universalgelehrte Alexander von Humboldt, der zu Beginn des 19. Jahrhunderts Landschaft als „Totalcharakter einer Gegend" bezeichnet.

1905 hält der Begriff Landschaft erstmalig Einzug in ein populäres Nachschlagewerk. In Meyers Großem Konversationslexikon wird Landschaft folgendermaßen definiert: „Landschaft ist jeder Ausschnitt der Erdoberfläche, den wir von einem bestimmten Standort aus zu überblicken vermögen, bis im Horizont oder Gesichtskreis Erde und Himmel 
zusammenzustoßen scheinen. Jede Landschaft kann unter einem naturwissenschaftlichen, unter einem künstlerischen oder unter einem kulturgeschichtlichen Gesichtspunkte betrachtet werden." Diese Definition spiegelt auch eine, im Vergleich zu bisherigen Betrachtungen, komplexere wissenschaftliche Wahrnehmung von Landschaft im Sinne einer durch anthropogene Eingriffe aus der Natur geformten Kulturlandschaft.

In der Wissenschaft wird die Betrachtung und Definition von Landschaft von nun an stark durch die Disziplin der Geographie geprägt, wobei sich die Begriffe Landschaft und Kulturlandschaft parallel weiterentwickeln. Selbstverständlich spielt auch weiterhin die Darstellung der Landschaft in der Malerei eine Rolle, insbesondere im Verständnis von dem, was man landläufig mit Landschaft assoziiert, doch verliert diese nun zugunsten der Naturwissenschaften an Bedeutung. In der zweiten Hälfte des 20. Jahrhunderts erhält die Diskussion einen neuen Impuls u. a. durch die Leipzig-Dresdener Schule der Landschaftsökologie, die zur Entwicklung eines systemorientierten Begriffs führte, der die naturräumliche Ausstattung mit dem Wirken des Menschen verknüpft.

Ende der 80er Jahre des 20. Jahrhunderts erlangte der Begriff der „historischen Kulturlandschaft“ als eine durch die kultivierende Tätigkeit des Menschen geprägte, besonders schützens- und bewahrenswerte Landschaft an Bedeutung. Hier sei insbesondere Hans Hermann Wöbse als Vorreiter genannt, der vor allem das ökologische Potenzial einer Landschaft betont. Noch immer gilt das Selbstverständnis, dass (Kultur-)Landschaft etwas Positives, Ästhe- tisches und vordergründig Schönes ist. Diese Assoziation wird jedoch in der zweiten Hälfte des 20. Jahrhunderts erstmals durch John Brinckerhoff Jackson hinterfragt, der mit seiner Ansicht, dass in Landschaften auch das „Hässliche und Temporäre“ zu sehen sei, zweifelsohne eine völlig neue Perspektive in der wissenschaftlichen (Kultur-)Landschaftsbetrachtung eröffnet hat.

Durch das im Jahr 2006 von der Ministerkonferenz für Raumordnung entwickelte Leitbild „Ressourcen bewahren, Kulturlandschaft gestalten" wird der Fokus auf eine aktive Gestaltung von Kulturlandschaften gelenkt. Vor diesem Hintergrund scheint eine erneute Befassung mit dem Begriff der Kulturlandschaft notwendig. In den folgenden Jahren gab es zahlreiche wissenschaftliche Auseinandersetzungen um eine „zeitgemäße“ Interpretation gegenwärtiger (Kultur-)Landschaften, so beispielsweise auch mehrere Arbeitskreise der Akademie für Raumforschung und Landesplanung.

Das dem vorliegenden Schwerpunktheft zugrunde liegende Projekt „Die gesellschaftliche Konstituierung von Kulturlandschaft" stellt sich die Frage: Wie konstituieren sich Kulturlandschaften durch subjektive Bedeutungszuschreibungen im alltagssprachlichen Handeln, in Diskursen und Diskurskoalitionen sowie durch die Koordination sektoraler Institutionensysteme und durch (Kultur-)Landschaftsverständnisse in der Landschaftsplanung aus soziologischer, politikwissenschaftlicher, planerischer und sozialgeographischer Perspektive?

Wir wünschen den Leserinnen und Lesern eine angenehme Lektüre! 